\title{
Unexpected genetic heterogeneity in a large consanguineous Brazilian pedigree presenting deafness
}

Karina Lezirovitz ${ }^{* 1}$, Eliete Pardono $^{1}$, Maria TB de Mello Auricchio ${ }^{1}$, Fernando L de Carvalho e Silva ${ }^{2}$, Juliana J Lopes ${ }^{2}$, Ronaldo S Abreu-Silva ${ }^{1}$, Jihane Romanos ${ }^{1}$, Ana C Batissoco ${ }^{1}$ and Regina C Mingroni-Netto ${ }^{1}$

${ }^{1}$ Centro de Estudos do Genoma Humano, Departamento de Genética e Biologia Evolutiva, Instituto de Biociências, Universidade de São Paulo, São Paulo, Brazil; ${ }^{2}$ Divisão de Educação e Reabilitação de Distúrbios da Comunicação (DERDIC), Pontifícia Universidade Católica, São Paulo, Brazil

Nonsyndromic autosomal recessive deafness accounts for $\mathbf{8 0} \%$ of hereditary deafness. To date, 52 loci responsible for autosomal recessive deafness have been mapped and 24 genes identified. Here, we report a large inbred Brazilian pedigree with 26 subjects affected by prelingual deafness. Given the extensive consanguinity found in this pedigree, the most probable pattern of inheritance is autosomal recessive. However, our linkage and mutational analysis revealed, instead of an expected homozygous mutation in a single gene, two different mutant alleles and a possible third undetected mutant allele in the MYO15A gene (DFNB3 locus), as well as evidence for other causes for deafness in the same pedigree. Among the 26 affected subjects, 15 were homozygous for the novel c.10573delA mutation in the MY015A gene, 5 were compound heterozygous for the mutation c.10573delA and the novel deletion c.9957_9960delTGAC and one inherited only a single c.10573delA mutant allele, while the other one could not be identified. Given the extensive consanguinity of the pedigree, there might be at least one more deafness locus segregating to explain the condition in some of the subjects whose deafness is not clearly associated with MYO15A mutations, although overlooked environmental causes could not be ruled out. Our findings illustrate a high level of etiological heterogeneity for deafness in the family and highlight some of the pitfalls of genetic analysis of large genes in extended pedigrees, when homozygosity for a single mutant allele is expected. European Journal of Human Genetics (2008) 16, 89-96; doi:10.1038/sj.ejhg.5201917; published online 12 September 2007

Keywords: autosomal recessive deafness; DFNB3 locus; MYO15A gene

\section{Introduction}

Hereditary hearing impairment is genetically heterogeneous, exhibiting patterns of inheritance that include: autosomal recessive, autosomal dominant, X-linked and

*Correspondence: Dr K Lezirovitz, Departmento de Genética e Biologia Evolutiva, Instituto de Biociências, Universidade de São Paulo, C.P. 11461, São Paulo, SP 05422-970, Brazil.

Tel: +55 113091 7478; Fax: +55 113091 7478;

E-mail: littlefelis@rocketmail.com

Received 11 April 2007; revised 2 August 2007; accepted 2 August 2007; published online 12 September 2007 mitochondrial. Nonsyndromic deafness represents 70\% of all cases. Nonsyndromic autosomal recessive deafness accounts for up to $80 \%$ of nonsyndromic hereditary hearing loss. To date, 52 loci responsible for this form of deafness have been mapped and 24 genes identified. ${ }^{1-4}$ Among all DFNB loci, the most prevalent one is DFNB1 (GJB2 and GJB6 genes, ${ }^{5-6}$ MIM 121011 and 604418) that accounts for up to $50 \%$ of recessive cases. Other loci with a significant number of different families associated are DFNB4 (SLC26A4 gene, ${ }^{7}$ MIM 605646), DFNB9 (OTOF gene, $^{8}$ MIM 603681), DFNB12 (CDH23 gene, ${ }^{9}$ MIM 
605516), DFNB7/11 (TMC1 gene, ${ }^{10}$ MIM 606706), followed by DFNB8/10 (TMPRSS3 gene, ${ }^{11}$ MIM 605511) and DFNB3 (MYO15A gene, ${ }^{12}$ MIM 602666).

Here, we report the study of a large inbred Brazilian pedigree with 26 individuals affected by prelingual deafness and presumed autosomal recessive inheritance to map the responsible gene. Instead of an expected homozygous mutation, we found two different novel MYO15A mutations segregating and evidence for other genetic and/or environmental causes for deafness in the same pedigree. Our findings illustrate the high level of genetic heterogeneity in deafness and the pitfalls of genetic linkage analysis in large pedigrees.

\section{Patients and methods Subjects}

The proband was referred to our genetic counseling service (Centro de Estudos do Genoma Humano, State of São Paulo, Brazil) by the clinical and educational institution DERDIC (Divisão de Educação e Reabilitação de Distúrbios da Comunicação, Pontifícia Universidade Católica de São Paulo). The parents of the proband were referred as nonconsanguineous, but his mother belonged to a large multigenerational inbred family living in a remote small village located in the countryside of a small town in the Northeast of Brazil. According to the local health care authorities, the small village had about 815 people, of whom 33 were affected by hearing impairment. The ascertained pedigree included 26 individuals affected by prelingual hearing loss (Figure 1).

\section{Audiological evaluation}

After performing otoscopy, rinoscopy and oroscopy exams, pure tone audiometry was carried out to test for air conduction $(250-8000 \mathrm{~Hz})$ and bone conduction (250$4000 \mathrm{~Hz}$ ). Pure tone audiometry was not performed in three of the 26 deaf subjects due to mental impairment (VI:4, VI:17 and VI:19).

\section{Ethical approval of study}

This study was approved by CONEP, the Brazilian National Committee on Ethics in Research. After appropriate written informed consents were obtained, blood samples were collected from 59 family members (26 affected).

\section{Molecular genetic analysis}

DNA was extracted by standard techniques using Kits such as 'Easy-DNA ${ }^{\mathrm{TM}}$ Kit (Version D) Genomic DNA Isolation' from Invitrogen (Carlsbad, CA, USA), a GFX Genomic Blood DNA Purification Kit (Amersham Biosciences, Buckinghamshire, UK) or using an equipment Autopure LS (Gentra Systems, Minneapolis, MN, USA).

\section{Screening for common deafness mutations}

The c.35delG mutation (GJB2 gene) was detected by allelespecific PCR as described by Scott et al. ${ }^{13}$ Sequencing of the coding exon of the GJB2 gene was performed in patients V:7, VI:17, VI:19, VI:3, VI:4 and VIII:1. Two fragments were amplified to sequence the entire coding exon. The first fragment was amplified with primers $1 \mathrm{~F}-5^{\prime}$-GTGTTGT GTGCATTCGTCTTTTC-3' and 3R - 5'-ACCTTCTGGGTTTT GATCTCCTC-3', and the second with primers $4 \mathrm{~F}-5^{\prime}$-GGA AGTTCATCAAGGGGGAGATA- $3^{\prime}$ and $2 \mathrm{R}-5^{\prime}$-CCTCATCCC TCTCATGCTGTCTA-3'. The $\operatorname{del}(G J B 6-\mathrm{D} 13 S 1830)$ and the $\operatorname{del}(G J B 6-D 13 S 1854)$ mutations in the GJB6 gene were tested by amplifying the breakpoint-containing fragments as described by del Castillo et al. ${ }^{14}$ The A1555G mutation was screened according to the protocol of Estivill et al. ${ }^{15}$

\section{Linkage analysis}

A genome-wide screening was performed with 382 microsatellite markers distributed with an average spacing of $10 \mathrm{cM}$ (ABI Prism Linkage Mapping Set 2.5, Applied Biosystems, Foster City, CA, USA). Additional microsatellite markers (D17S805, D17S2196, D17S783, D17S1873, D17S2206 and D17S2207) within the DFNB3 chromosomal region were also amplified using fluorescent-labeled primers. All fluorescent-labeled PCR products were analyzed in the MegaBACE 1000 DNA Analysis System with the software Genetic Profiler version 2.2 (Amersham Biosciences).

\section{MYO15A gene analysis}

The exons of the MYO15A gene were sequenced using the DYEnamic ET Dye Terminator Cycle Sequencing Kit (Amersham Biosciences) and analyzed in the MegaBACE 1000 DNA Analysis System (Amersham Biosciences). The primers' sequences to amplify all exons of the MYO15A gene were suggested by Dr Thomas Friedman. On average, about 20 nt flanking coding sequences were examined. Nomenclature of the mutations was adapted to follow the recommendations of den Dunnen and Antonarakis. ${ }^{16}$ Accordingly, nucleotide 1 is the A of the ATG-translation initiation codon in GenBank accession number AF144094. For mutation description in the protein level, the GenBank accession number NP_05723 was used.

Figure 1 Pedigree showing the haplotypes of the DFNB3 chromosomal region. The black and the gray haplotypes segregate with deafness. Subjects from branch 1 are compound heterozygous for mutations c.10573delA/c.9957_9960delTGAC in the MYO15A gene; subjects from branch 2 are homozygous for c. 10573 delA mutation, the exception is subject VII:2 (*) who is a compound heterozygote, like subjects from branch 1 . Subjects from branches 3 and 4 did not present any of these mutations. 


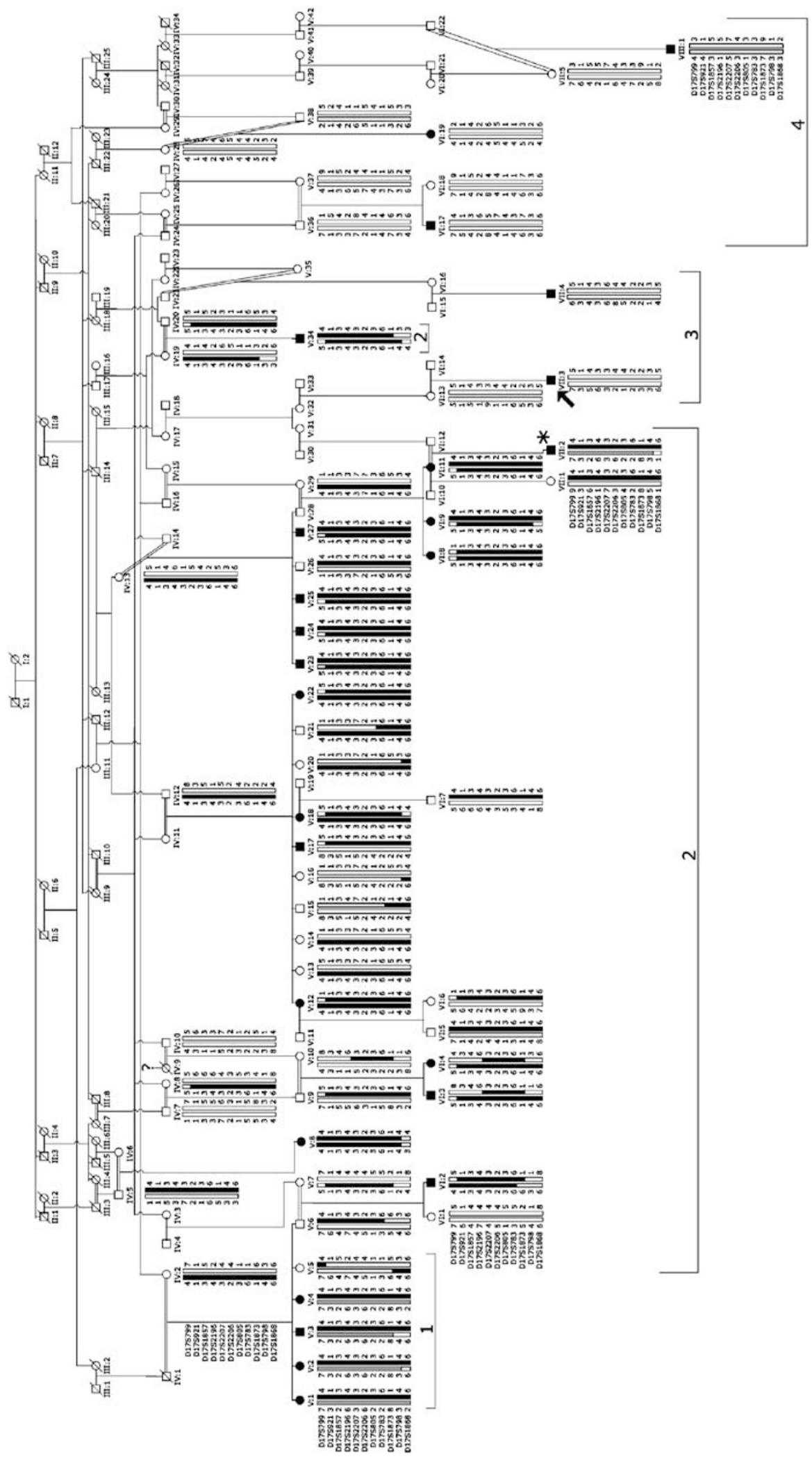


To characterize the frameshift mutation found in exon 62 (MYO15A gene) in samples from patients $\mathrm{V}: 1, \mathrm{~V}: 2, \mathrm{~V}: 3$, $\mathrm{V}: 4$ and VII:2 cloning of the PCR products was performed using pGEM-T Easy Vector Systems I (Promega) kit before sequencing.

\section{Lod score calculations}

Lod scores were calculated by using MLINK of the FASTLINK computer package. ${ }^{17}$ The disease was coded as fully penetrant while the disease allele frequency was set at 0.0001 in compliance with published studies. Meiotic recombination frequencies were considered to be equal for male and female subjects. Allele frequencies for the microsatellite markers were set to be $1 / \mathrm{N}, \mathrm{N}$ being the number of different alleles found in the family.

\section{Results and discussion}

We studied 26 individuals affected by prelingual hearing loss belonging to the same consanguineous family that lived in a small remote rural village (Figure 1). From the 26 affected subjects, 24 were born from 12 consanguineous marriages. Consanguinity was not documented for the parents of the proband (VII:3) and for the parents of the subject VII:4. Out of the 12 consanguineous marriages, 11 shared a common ancestral couple (I:1 and I:2). However, consanguinity between the individuals IV:1 and IV:2 (branch 1 from Figure 1) could not be related to the couple I:1 and I:2, but was due to the common ancestors II:5 and II:6.

In most of the audiometric evaluated subjects (22 of 23), deafness was sensorineural, severe to profound with a prelingual onset (Figure 2a). One subject (VIII:1) had mixed moderate hearing impairment also with a prelingual onset (Figure 2b). No other abnormalities were observed in these 23 subjects. The remaining three affected individuals also had mental impairment, which prevented the conduction of the pure tone audiometry (VI:4, VI:17 and VI:19), therefore, we could not characterize their deafness.

Based on the extensive consanguinity displayed by this family, we assumed that the pattern of inheritance was autosomal recessive and that a single deafness locus should be segregating in the pedigree, explaining all cases of deafness. Extended pedigrees with high number of a

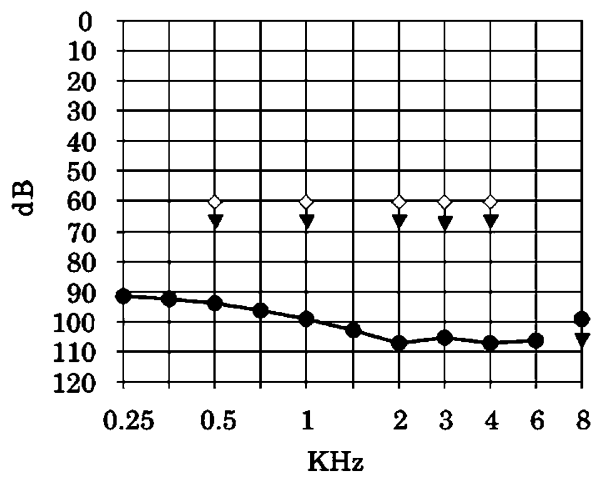

- air conduction $\diamond$ bone conduction

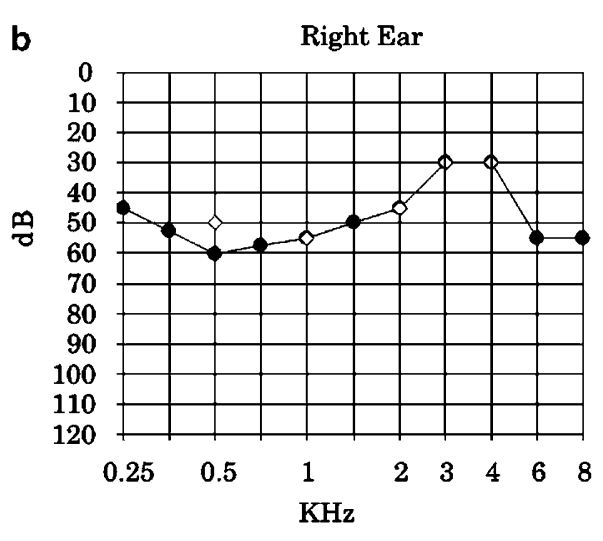

$-\infty$ air conduction $\diamond$ bone conduction

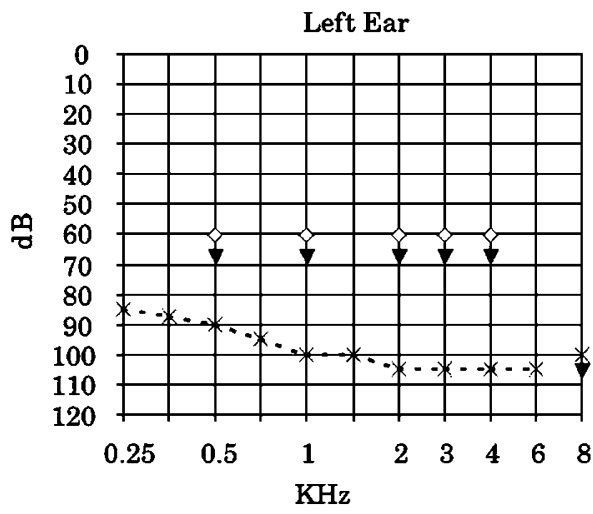

$-\cdot \rtimes \cdot$ air conduction $\diamond$ bone conduction

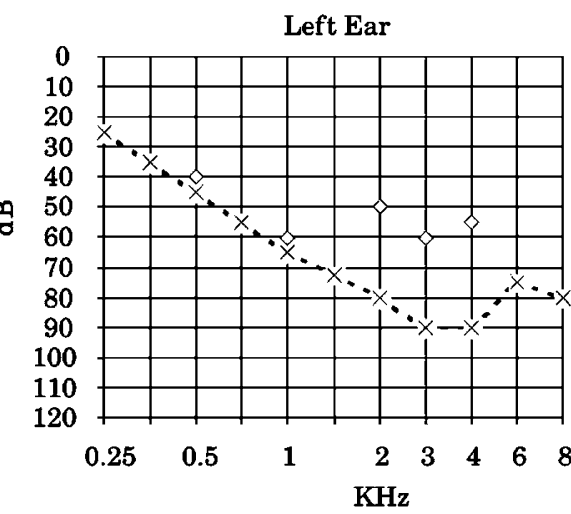

- - $\times$ - air conduction $\diamond$ bone conduction

Figure 2 (a) Tonal audiometry showing the average thresholds of the 22 subjects with sensorineural severe to profound hearing impairment; (b) tonal audiometry of patient VIII:1 showing mixed hearing loss. 
consanguineous marriages are considered powerful tools to map new loci through homozygosity mapping because of the expected genetic homogeneity.

After ruling out frequent causes of genetic deafness, such as mutations in the GJB2 gene, the two deletions (delGJB6(D13S1830) and delGJB6(D13S1854)) involving the GJB6 gene and the A1555G mitochondrial mutation, a genome-wide scan was performed. However, the genomewide scan results did not allow us to find a common region of linkage and/or region of homozygosity for all 26 deaf subjects. While accounting for the possibility of genetic heterogeneity within the pedigree, the most suggestive candidate region to explain deafness in the majority of the affected individuals appeared to be the DFNB3 locus (black and gray haplotypes in Figure 1). Segregation analysis of the haplotypes showed that 15 of the 26 affected subjects were homozygotes for the DFNB3 chromosomal region (black haplotypes displayed in branch 2 of Figure 1). This haplotype analysis also suggested that the four affected sibs (V:1, V:2, V:3 and V:4) from branch 1 (Figure 1), whose parental consanguinity was not related to the same ancestral couple from branches 2 and 4, were compound heterozygotes for mutations in the DFNB3 gene (black and gray haplotypes in Figure 1). Another deaf subject belonging to branch 2 (VII:2, * of Figure 1), also appeared to be a compound heterozygote for mutations in the DFNB3 gene. Furthermore, there was one subject who inherited only one of the risk haplotypes (V:17) and five subjects, including the proband, who did not inherit any of the risk haplotypes (VI:17, VI:19,VII:3, VII:4 and VIII:1).

Lod score calculations were performed excluding all five subjects with none of the risk haplotypes and omitting some of the inbreeding loops due to software limitations. The highest two-point lod scores, 12.1 for $\theta=0.01$ and 10.81 for $\theta=0.01$, were obtained with markers D17S805 ( $\sim 1 \mathrm{Mb}$ apart from the MYO15A gene) and D17S2207 (D17S2207 being located inside the MYO15A gene, intron $1-2)$, respectively.

In the 15 subjects that were homozygous for a haplotype in the DFNB3 chromosomal region (branch 2 in Figure 2), we found a novel frameshift mutation c.10573delA in the last exon of the MYO15A gene (Figure $3 \mathrm{a}$ and b). This mutation would eliminate the last six amino acids at the C-terminus of myosin-XVa, which includes the four aminoacid long class 1 PDZ binding ligand, and would abolish the natural stop codon leading to the addition of 28 novel amino acids to the protein (p.Ser3525fs). In the five subjects whose haplotype analysis indicated two different mutations, we found another novel frameshift mutation c.9957_9960delTGAC in exon 62, in addition to the c.10573delA in exon 66 (Figure 3c and d). This mutation would lead to a premature stop codon in the second novel codon caused by the frameshift (p.Asp3320fs), thus it would eliminate 211 amino acids of the C-terminus of myosin XVA, which include approximately half of the second FERM domain and the entire C-terminal class 1 PDZ ligand. We concluded that those mutations were pathogenic and were the cause of deafness in the 20 out of the 26 deaf subjects ascertained from the pedigree (Table 1). Thus, we confirmed the hypothesis that patients from branch 1 were compound heterozygotes for mutations in the MYO15A gene. Although their parents were first cousins, their deafness was not related to the consanguinity but to two different MYO15A mutations. To try to find out the frequency of those mutations in a deaf Brazilian population, we screened both mutations in a cohort of 70 deaf probands ascertained in our Genetic Counseling Service whose family history indicated an autosomal recessive inheritance. But none was detected.

The DFNB3 locus was first mapped to chromosome region 17p11.2 in a kindred from an isolated village called Bengkala in Bali, segregating autosomal recessive prelingual deafness. ${ }^{18}$ Subsequently, Wang et al ${ }^{19}$ found three different myosin-XV (MYO15A) mutations segregating with profound congenital deafness, one in the inbred Bengkala kindred and the others in two unrelated consanguineous families from India. In 2001, Liburd et al ${ }^{20}$ described three consanguineous families from Pakistan with homozygous missense MYO15A mutations causing deafness as well as one deaf Smith-Magenis's syndrome (del(17)p11.2) patient who was hemizygous for a missense mutation of $M Y O 15 A$. Nal et al ${ }^{12}$ described 16 novel mutations in the MYO15A gene associated with deafness in families from Pakistan, India and Turkey. It is noteworthy that almost all families described with MYO15A mutations were consanguineous, one as large as the Brazilian (the Bengkala kindred) and 25 smaller ones, with only a single mutant allele as expected. The high number of exons of MYO15A, which discourage its mutational screening, might explain the few number of reports on MYO15A mutations. The MYO15A gene has 66 exons and its longest mRNA has $11,876 \mathrm{nt}$ (AF144094). Myosin-XVa is a motor protein that uses energy from ATP hydrolysis to move along actin filaments. It is localized at the tips of hair cell stereocilia. ${ }^{21-23}$ As indicated by the study of Belyantseva et $a{ }^{22}$ myosin-XVa might be the only motor in hair cells that selectively deliver cargo to the tips of stereocilia. Whirlin is a multi-PDZ domain-containing protein and its delivery by myosin-XVa to stereocilia tips is essential for stereocilia elongation and for the staircase architecture of the hair bundle. Both the c.10573delA and the c.9957_9960delTGAC novel mutations found in the Brazilian family lie in exons of MYO15A predicted to encode its tail domains and they probably eliminated the ability of myosin-XVa to bind and transport whirlin to the tips of stereocilia. ${ }^{22}$ Belyantseva et $a l^{22}$ showed that the C-terminal class 1 PDZ ligand of myosin-XVa interacts with the third PDZ domain of whirlin. Thus, elimination of either module would result in a failure of these two proteins to interact. 

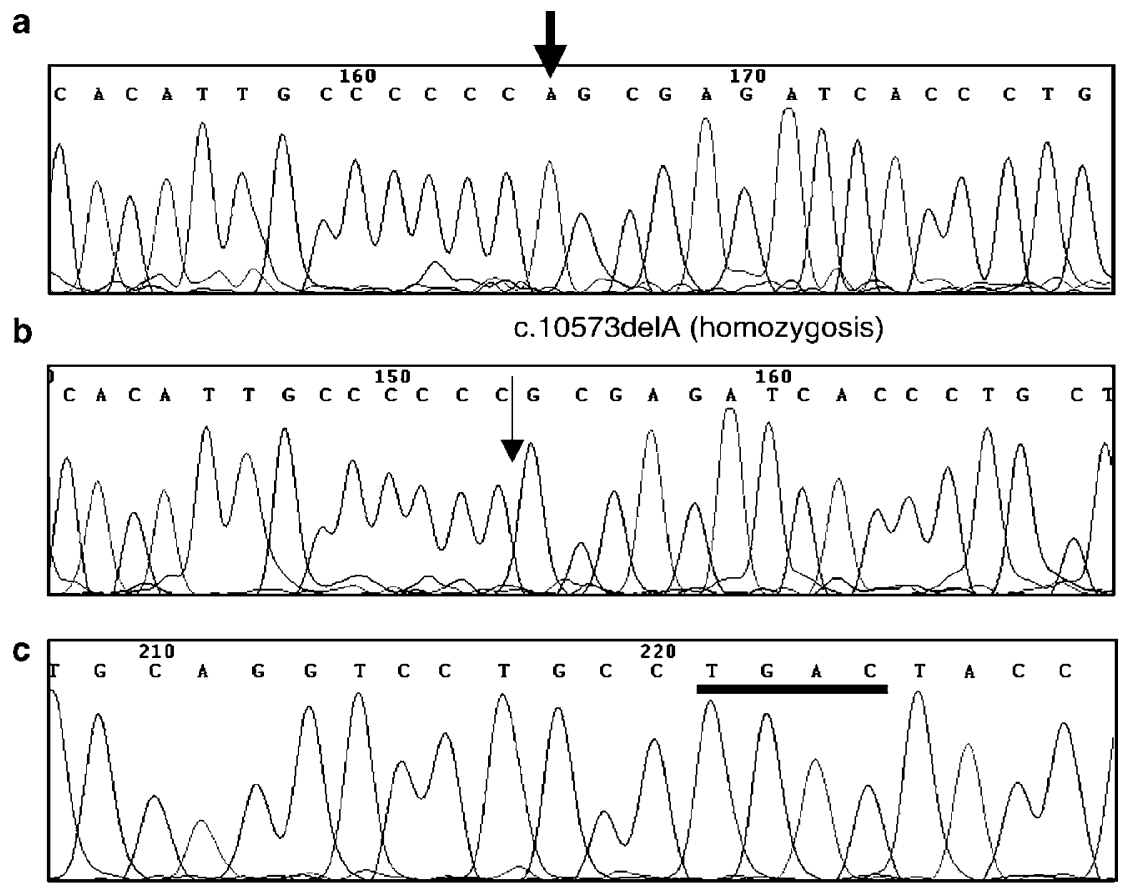

d c.9957-9960delTGAC (subcloned allele)

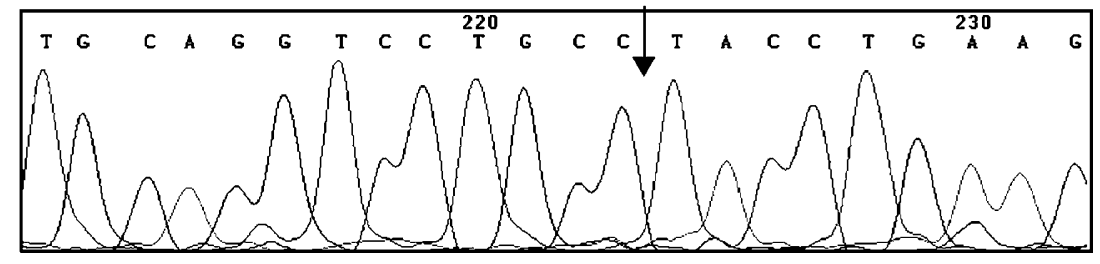

Figure 3 Partial sequence of exon 66 of the MY015A gene from (a) a control sample, (b) a sample bearing the c.10573delA mutation in homozygosis. Partial sequence of exon 62 of the MYO15A gene from patient (VII:2)'s (c) subcloned normal allele and (d) subcloned mutant allele bearing the c.9957_9960delTGAC mutation.

Table 1 Summary description of the genotypic data

\begin{tabular}{lcc}
\hline Genotypic data & $\begin{array}{c}\text { Number of } \\
\text { individuals }\end{array}$ & Pedigree position \\
\hline MYO15A mutations in both alleles & 20 & \\
C.10574delA homozygotes & 15 & $\mathrm{~V}: 8, \mathrm{~V}: 12, \mathrm{~V}: 18, \mathrm{~V}: 22, \mathrm{~V}: 23, \mathrm{~V}: 24, \mathrm{~V}: 25, \mathrm{~V}: 27, \mathrm{Vl}$ \\
& & $\mathrm{V}: 34, \mathrm{Vl}: 1, \mathrm{Vl}: 3, \mathrm{Vl}: 4, \mathrm{Vl}: 8, \mathrm{VI}: 9$ and VI:11 \\
$\quad$ C.10574delA/c.9958_9961delTGAC compound heterozygotes & 5 & $\mathrm{~V}: 1, \mathrm{~V}: 2, \mathrm{~V}: 3, \mathrm{~V}: 4$ and VII:2 \\
Unsolved cases & 6 & \\
$\quad$ One MYO15A mutation detected & 1 & $\mathrm{~V}: 17$ \\
$\quad$ No MYO15A mutations & 5 & $\mathrm{VI}: 17, \mathrm{Vl}: 19, \mathrm{VII}: 4, \mathrm{VII}: 3$ and VIII:1 \\
Total & 26 & \\
\hline
\end{tabular}

The subject V:17 (branch 2 of Figure 1), who presented sensorineural severe to profound deafness like his siblings, inherited only one of the risk haplotypes. Indeed, he carried only the c.10573delA allele as a single copy. Sequencing of the exons as well as exon-intron boundaries did not allow us to identify a second mutation. It is possible that a mutation in a regulatory element or in the promoter region of MYO15A is present in his other allele. Given the low social economic profile of the region and lack of health assistance, the hypothesis of environmental causes for deafness cannot be excluded completely. Besides, as the individual was born from a first-cousin marriage, 
the possibility of another recessive locus explaining his deafness could also be considered.

There are five remaining deaf subjects whose haplotypes did not indicate that MYO15A gene as the causative one. Among them, three (VI:17, VI:19 and VIII:1) were born from consanguineous marriages and exhibited distinct clinical presentations: two had also mental impairment (VI:17 and VI:19) and one had mixed hearing loss (VIII:1) (branch 4 in Figure 1). Patient VI:17 showed a severe mental retardation associated with hyperactivity. Patient VI:19 was described as presenting learning difficulties. There are about 150 entries in the OMIM database, which describe syndromes that may associate mental retardation and deafness, and all of them were characterized by other physical abnormalities or facial dysmorphisms. Physical examination revealed no additional abnormalities and it was not possible to associate their clinical presentation to any known syndrome. With the exception of embryopathies induced by rubella, toxoplasmosis or cytomegalovirus infection, which might result in polymalformations including hearing impairment, most forms of syndromic hearing impairment are of genetic origin. ${ }^{2}$ It is possible that the association of deafness and mental retardation was caused by environmental factors. Nevertheless, given the parental consanguinity, the most likely explanation for their deafness and/or mental retardation are homozygous mutant alleles of different loci segregating in this pedigree. The proband (VII:3) and the subject VII:4 (both from Branch 3 in Figure 1) presented a similar phenotype to those subjects bearing MYO15A mutations and no other clinical signs or symptoms. The proband and the subject VII:4 shared a similar DFNB3 haplotype, with exception of marker D17S2207. The hypothesis of a deletion of marker D17S2207 was ruled out based on the genotype of the proband's mother. Thus, the fact that VII:3 and VII:4 presented a similar DFNB3 haplotype is most likely a coincidence. Genetic causes as well as overlooked environmental causes could explain their conditions. Mutations in the coding region of the GJB2 gene were excluded in the five deaf patients without risk haplotypes and in patient V:17 with only one mutation detected. Moreover, in all microsatellites close to known autosomal recessive deafness loci, the six subjects exhibited different genotypes, indicating that genes in those known regions could not explain deafness in all of them.

The success of homozygosity mapping relies on the assumption that, for a rare recessive disease, patients in a consanguineous family will be homozygous for a mutation derived from a common ancestor of the parents. This strategy was successfully used many times in mapping genes responsible for deafness. Although, deafness is a frequent disease, there are many different loci associated. With exception of mutations in the GJB2, PDS and CDH23 genes, reported mutations in each locus are relatively rare. Locus and allelic heterogeneity within the same consanguineous pedigree, represents one of the important pitfalls in the homozygosity mapping strategy. Studying only closely related individuals is commonly pointed out to avoid the pitfall of genetic heterogeneity. The results found in the Brazilian pedigree represent a departure from both expectations and there are other examples in the literature. Van Soest $e t a l^{24}$ described a genetic isolate population from the Netherlands in which two different loci responsible for autosomal recessive retinitis pigmentosa were segregating. De Brouwer et $a l^{25}$ studied a large family with many individuals affected by autosomal recessive nonsyndromic childhood hearing loss (DFNB). Failure to demonstrate linkage in a genome-wide scan has suggested genetic heterogeneity for the hearing loss in this family: patients in one branch of the family (branch C) were homozygous for the 35delG mutation in the GJB2 gene (DFNB1); patients from branch A were homozygous for a mutation in the $\mathrm{CDH} 23$ gene; patients in branch $\mathrm{B}$ were compound heterozygotes for the $\mathrm{CDH} 23$ gene mutation found in branch $\mathrm{A}$ and a second mutation. Fendri et $a l^{26}$ also described genetic heterogeneity within a consanguineous family involving two genes responsible for Sarcoglycanopathies (LGMD $2 D$ and $L G M D 2 C$ ). In 2000, Miano et $a l^{27}$ reported why their genome-wide linkage analysis failed to identify the chromosomal region associated with the enhanced S-cone syndrome in three consanguineous families. Later mutational analysis revealed homozygous mutations in two of the families, but in one family, three affected individuals were homozygous for a mutation and one was a compound heterozygote, with a yet unidentified mutation.

In summary, two different novel mutations in the MYO15A gene fully explain deafness in 20 out of 26 affected subjects in the Brazilian inbred pedigree (Table 1). In addition, it is possible that a further unrecognized MYO15A mutant allele is present in the subject with only one MYO15A mutation detected. The cause of the deafness in the remaining five subjects does not appear to be related to mutations in MYO15A and other unidentified loci may be involved, though environmental factors could not be fully excluded.

Finally, we conclude that at least two mutations in the MYO15A gene and other genetic and/or environmental factors caused deafness in this large consanguineous Brazilian pedigree.

\section{Acknowledgements}

We thank all family members for their enrollment in this study and laboratory fellows for their collaboration, especially Maria Cristina Celia Braga for proband ascertainment and Camila Juncansen for technical support. We also thank all professionals from DERDIC for their collaboration in the clinical evaluation of our patients and $\mathrm{Dr}$ Peter Pearson for critical reading of the manuscript. We also thank Dr Thomas Friedman for sending us the primers to amplify D17S2207 and D17S2206 markers and for providing the primer sequences to 
amplify all exons from the MYO15A gene. This work was financially supported by Fundação de Amparo à Pesquisa do Estado de São Paulo (FAPESP) - CEPID, and Conselho Nacional de Desenvolvimento Científico e Tecnológico (CNPq) - PRONEX.

\section{References}

1 van Camp G, Smith RJH: Hereditary Hearing Loss Homepage (07/2006), http://webhost.ua.ac.be/hhh/.

2 Petit C: From deafness genes to hearing mechanisms: harmony and counterpoint. Trends Mol Med 2006; 12: 57-64.

3 Petersen $\mathrm{MB}$, Willems PJ: Non-syndromic, autosomal recessive deafness. Clin Genet 2006; 69: 371-392.

4 Khan SY, Riazzudin S, Tariq M et al: Autosomal recessive nonsyndromic deafness locus DFNB63 at chromosome 11q13.2-q13.3. Hum Genet 2006; 120: 789-793.

5 Gasparini P, Rabionet R, Barbujani G et al: High carrier frequency of the 35delG deafness mutation in European populations. Genetic analysis consortium of GJB2 35delG. Eur J Hum Genet 2000; 8: 19-23.

6 Snoeckx RL, Huygen PLM, Feldmann D et al: GJB2 mutations and degree of hearing loss: a multicenter study. Am J Hum Genet 2005; 77: $945-957$.

$7 \mathrm{Hu} \mathrm{H}, \mathrm{Wu} \mathrm{L}$, Feng Y et al: Molecular analysis of hearing loss associated with enlarged vestibular aqueduct in the mainland Chinese: a unique SLC26A4 mutation spectrum. J Hum Genet 2007; 52: 492-497.

8 Varga R, Avenarius MR, Kelley PM et al: OTOF mutations revealed by genetic analysis of hearing loss families including a potential temperature sensitive auditory neuropathy allele. I Med Genet 2006; 43: 576-581.

9 Astuto LM, Bork JM, Weston MD et al: $\mathrm{CDH} 23$ mutation and phenotype heterogeneity: a profile of 107 diverse families with Usher syndrome and nonsyndromic deafness. Am J Hum Genet 2002; 71: 262-275

10 Kurima K, Peters LM, Yang Y et al: Dominant and recessive deafness caused by mutations of a novel gene, TMC1, required for cochlear hair-cell function. Nat Genet 2002; 30: 277-284.

11 Scott HS, Kudoh J, Watternhofer M et al: Insertion of beta-satellite repeats identifies a transmembrane protease causing both congenital and childhood onset autosomal recessive deafness. Nat Genet 2001; 27: 59-63.

12 Nal N, Ahmed ZM, Erkal E et al: Mutational spectrum of MYO15A: the large $\mathrm{N}$-terminal extension of myosin XVA is required for hearing. Hum Mutat 2007, June 1 [E-pub ahead print].

13 Scott DA, Kraft ML, Carmi R et al: Identification of mutations in the connexin 26 gene that cause autosomal recessive nonsyndromic hearing loss. Hum Mutat 1998; 11: 387-394.
14 del Castillo FJ, Rodríguez-Ballesteros M, Alvarez A et al: A novel deletion involving the connexin-30 gene, del(GJB6-D13S1854), found in trans with mutations in the GJB2 gene (connexin-26) in subjects with DFNB1 non-syndromic hearing impaiment. $J$ Med Genet 2005; 42: 588-594.

15 Estivill X, Govea N, Barceló E et al: Familial progressive sensorineural deafness is mainly due to the mtDNA A1555G mutation and is enhanced by treatment with aminoglycosides. Am J Hum Genet 1998; 62: 27-35.

16 den Dunnen JT, Antonarakis SE: Recommendations for the description of sequence variants, 2007, http://www.hgvs.org/ mutnomen/recs.html.

17 Cottingham Jr RW, Idury RM, Schäffer AA: Faster sequential genetic linkage computations. Am J Hum Genet 1993; 53: 252263.

18 Friedman TB, Liang Y, Weber JL et al: A gene for congenital, recessive deafness DFNB3 maps to the pericentromeric region of chromosome 17. Nat Genet 1995; 9: 86-91.

19 Wang A, Liang Y, Fridell RA et al: Association of unconventional myosin MYO15 mutations with human nonsyndromic deafness DFNB3. Science 1998; 280: 1447-1451.

20 Liburd N, Ghosh M, Riazuddin S et al: Novel mutations of MYO15A associated with profound deafness in consanguineous families and moderately severe hearing loss in a patient with Smith-Magenis syndrome. Hum Genet 2001; 109: 535-541.

21 Belyantseva IA, Boger ET, Friedman TB: Myosin XVa localizes to the tips of inner ear sensory cell stereocilia and is essential for staircase formation of the hair bundle. Proc Natl Acad Sci 2003; 100: $13958-13963$.

22 Belyantseva IA, Boger ET, Naz S et al: Myosin-XVa is required for tip localization of whirlin and differential elongation of hair-cell stereocilia. Nat Cell Biol 2005; 7: 148-156.

23 Stepanyan R, Belyantseva IA, Griffith AJ, Friedman TB, Frolenkov GI: Auditory mechanotransduction in the absence of functional myosin-XVa. J Physiol 2006; 576: 801-808.

24 van Soest S, van den Born LI, Gal A et al: Assignment of a gene for autosomal recessive retinitis pigmentosa (RP12) to chromosome $1 \mathrm{q} 31-\mathrm{q} 32.1$ in an inbred and genetically heterogeneous disease population. Genomics 1994; 22: 499-504.

25 de Brouwer APM, Pennings RJE, Roeters $\mathrm{M}$ et al: Mutations in the calcium-binding motifs of $\mathrm{CDH} 23$ and the 35delG mutation in GJB2 cause hearing loss in one family. Hum Genet 2003; 112: $156-163$.

26 Fendri K, Kefi M, Hentati F, Amouri R: Genetic heterogeneity within a consanguineous family involving the LGMD 2D and the LGMD 2C genes. Neuromuscul Disord 2006; 16: 316-320.

27 Miano MG, Jacobson SG, Carothers A et al: Pitfalls in homozygosity mapping. Am J Hum Genet 2000; 67: 1348-1351. 